\title{
Incidence of dentinal crack formation during root canal preparation with two NiTi instruments activated by adaptive motion and continuous rotation: an in vitro study
}

\author{
Erhan Erkan๑, Keziban Olcay๑, Tan Fırat Eyüboğlu๑, Mustafa Gündoğarø \\ Department of Endodontics, İstanbul Medipol University School of Dentistry, Ístanbul, Turkey
}

DOI: 10.18621 eurj.417982

\begin{abstract}
Objectives: The aim of this study was to evaluate dentin crack incidence after root canal instrumentation using ProTaper Next (PT Next; Dentsply Maillefer, Ballaigues, Switzerland) and Twisted File Adaptive (TF Adaptive; Sybron Endo, Orange, California, USA) at different kinematics.

Methods: Sixty human extracted premolar teeth were selected and divided into 4 main groups. Group 1: PT Next with continuous rotation $(n=15)$; Group 2: PT Next with adaptive motion $(n=15)$; Group 3: TF Adaptive with continuous rotation $(n=15)$; Group 4: TF Adaptive with adaptive motion $(n=15)$. Fifteen unprepared teeth were used as control group. Crowns of the teeth were removed and roots were sectioned at 3, 6, and 9 $\mathrm{mm}$ from the apex using a diamond saw. Finally root dentin pieces were evaluated under stereomicroscope at x 25 magnification. Digital images were evaluated by 2 researchers.

Results: No cracks were observed in the control group. In groups 2 and 4 no fracture was observed at the level of $9 \mathrm{~mm}$ and similarly in group 4 at $6 \mathrm{~mm}$ level. Group 3 showed a significantly higher dentin crack formation followed by Group 1, 2, 4 and control group respectively $(p<0.039)$. There was no significant difference between groups at $6 \mathrm{~mm}$ and $9 \mathrm{~mm}$ levels $(p=0.497)$ except for only $3 \mathrm{~mm}$ level $(p<0.035)$.

Conclusions: It was concluded, both adaptive motion and continuous rotationpromoted dentinal defect. Adaptive motion produced less dentinal defects all dentin levels but there was no significant difference.

Keywords: Dentinal crack, root canal preparation, protaper next, twisted file adaptive, nickel-titanium instruments, adaptive motion, continuous rotation
\end{abstract}

Received: April 24, 2018; Accepted: December 27, 2018; Published Online: June 30, 2019

$\mathrm{T}$ he main goal of the root canal enlargement is to provide the biomechanical cleaning and shaping of the root canal dentin [1]. Stainless steel hand instruments may cause iatrogenic damages such as canal transportations, ledge formation and perforations especially in curved root canals [2]. To avoid these damages, Nickel-Titanium (NiTi) rotary instruments are the most preferred filesbydentists during the root canal shaping because of its shape memory and super elasticity properties $[3,4]$. Usage of these tools has many advantages in endodontic treatment, such as shortening the working time and increasing the quality of irrigation too [1].

On the other hand the amount of remaining dentin volume is very important for strength of a root filled tooth [5]. Dentine removal during root canal shaping

Address for correspondence: Erhan Erkan, Assistant Professor, İstanbul Medipol University School of Dentistry, Department Endodontics, TEM Avrupa Otoyolu, Göztepe Çıkışı, No:1, Băgcılar 34214, İstanbul, Turkey

E-mail: eerkan@medipol.edu.tr 
with NiTi rotary instruments may create crack formation or vertical root fracturesbecause of the aggressive cutting ability and tapered file design $[6,7]$. NiTi instruments can be used at two different movements: Continuous rotation or Reciprocal motion. In addition alternative motion has been introduced in the recent years called as "Adaptive Motion" which uses a combination of continuous rotation and reciprocal motion cinematics.

Root canal shaping movement with NiTi rotary systems may create dentinal damages to various degrees [1]. In addition torsional and flexural-related cracks due to shaping NiTi can occur at continuous rotation. On the other hand reciprocal motion reduces stress on the NiTi rotary instruments and help to reduce cyclic fatigue fracture [8]. But reciprocal motion decreased cutting efficiency of NiTi instruments because of the clockwise (CW/counterclockwise (CCW) movement at small or equal degrees. It means that more apical pressure of the files may create dentinal cracks [9]. But Liu et al. [10] reported that reciprocal motion creates less dentinal crack formationthan continuous rotation. However Bürklein et al. [8] study showed more dentinal crack formation in reciprocation movement in the apical root area and Karataş et al. [11] reported that dentinal micro-cracks formation ratio is dramatically increased with reciprocal motion compared to continuous rotation. There is no consensus on dentinal crack formation about NiTi rotary instruments in root canal shaping.

NiTi files with adaptive motion can change the movement from continuous rotation to reciprocal mode with $\mathrm{CW}$ and $\mathrm{CCW}$ angles varying from $0^{\circ}$ to $600^{\circ}$ up to $370-50^{\circ}$ at high pressure values. The purpose of this technology is to allow the TF Adaptive file to adjust to the intra-canal torsional forces, depending on the amount of pressure placed in the file (TF Adaptive Brochure). So this movement seems to have the advantages of both technics.

Therefore, the aim of the present study was to investigate the effect of different kinematics (adaptive motion and continuous rotation) using two tested $\mathrm{NiTi}$ rotary systems (PT Next; Dentsply Maillefer, Ballaigues, Switzerland and TF Adaptive; TF Adaptive; Sybron Endo, Orange, California, USA) on dentinal crack formation. The tested null hypothesis was that there would be no significant differencesin the incidence of dentinal crack formation between the groups.

\section{METHODS}

The research protocol of this study was approved by the Istanbul Medipol University Ethical Board of Clinical Trials and Non-Interventional Research (Approval Number: 10840098-604.01.01-E.482). Freshly extracted, intact, non-carious, human mandibular premolar teeth with mature apices and straights roots $\left(<25^{\circ}\right)$ were selected for the study. The single-rooted teeth with single root canal formation are our first criteria for the present study. In addition, the cross-sectional anatomy anatomies of the lower premolar teeth were oval in the coronal third of the root and take round form towards to the tooth apex in all samples. Figure 1 showed only craze-lines and nodefects samples from different areas of the tooth which were chosen randomly. Therefore, due to this randomization some of the images included coronal third of the root canals from different samples whilst the others did not. Teeth were stored in distilled water and single canal formation was controlled by radiographs before starting the experiment.All of the samples were cleaned with water and the soft tissue and calculus were debrided mechanically. All roots were controlled with under $\times 45$ magnification using a stereomicroscope (Carl Zeiss Axio Zoom V16, Jena, Germany)for the integrity of the root structure. The teeth with cracks on the root surface were not included in the study and replaced with solid teeth. The sample size was calculated as 15 in each group, a type I error of 0.05 , and a statistical power of $80 \%$ using the $G^{*}$ Power software (version 3.1.9.2).

Finally 75 teeth were selected for the study according the all criteria.All crowns were removed using $0.19 \mathrm{~mm}$ thick diamond saw (Horico SH 394C190) under water cooling from the cementoenamel junction all root sample length was equalized at $15 \mathrm{~mm}$.Root canal lengths were measured by \#15 file inserted into the canal until the tip of the file became visible at the apical foramen. Working length (WL) wasdetermined by subtracting $1 \mathrm{~mm}$ from the root canal length. Apical patency was also controlled by \#10 K-file during the root canal preparation.

Teeth were randomly divided to fourexperimental group and one control group $(n=15)$. PT Next NiTi Rotary System (Dentsply Maillefer, Ballaigues, Switzerland) and TF Adaptive NiTi Rotary System (SybronEndo, Orange, California, USA) were used 
with different kinematics in the present study:

Group 1: PT Next with Continuous Rotation

Group 2: PT Next with Adaptive Motion

Group 3: TF Adaptive with Continuous Rotation

Group 4: TF Adaptive with Adaptive Motion

Root canal instrumentation with adaptive motion was achieved by Elements Motor (SybronEndo, Orange, California, USA) and continuous rotation was achieved by X-smart Plus Endodontic Motor (Dentsply Maillefer, Ballaigues, Switzerland). The NiTi files at Group 1 and Group 4 were used at recommended values of torque and speed according to the manufacturers' instructions. After each instrument insertion, the teeth were irrigated with 10 $\mathrm{ml}$ of 2, 6\% $\mathrm{NaOCl}$ solution (Canal Pro ${ }^{\mathrm{TM}}$ Coltene/Whaledent, Switzerland) with 30 gauge irrigation needle (Canal Clean, Biodent Co. South Korea) was used the irrigation solution at the all groups:

\section{Group 1: PT Next with Continuous Rotation}

The root canals were prepared using X-Smart Plus (Dentsply Maillefer) at $300 \mathrm{rpm}$ with a torque $2 \mathrm{Ncm}$ with in-out motion for all files. X1 (size 17, 0,04 taper) and X2 (size 25, 0,06 taper) was used at full WL.

Group 2: PT Next with Adaptive Motion

Instrumentation was achieved by Elements Motor (Sybron Endo, Orange, California, USA) with "Adaptive Motion" mode. X1 and X2 was used at full WL.

Group 3: TF Adaptive with Continuous Rotation Instrumentation was achieved with Elements Motor (Sybron Endo, Orange, California, USA) by $500 \mathrm{rpm}$ and the torque at $2 \mathrm{Ncm}$. SM1 (size 20; 0,04 taper) and SM2 (size 25; 0,06 taper) was used at working length.It was finished at SM2 (size 25; 0,06 taper).

Group 4: TF Adaptive with Adaptive Motion

Root canal preparation was achieved with Elements Motor (Sybron Endo, Orange, California, USA) by adaptive mode. SM1) and SM2 were used at working length. It was finished at SM2.

All preparations were achieved by one operator and two operators who were blinded examined the crack classification. The samples sectioned horizontally at 3, 6 and $9 \mathrm{~mm}$ from the apex with 0 , $19 \mathrm{~mm}$ diamond saw (Horico SH 394C190)under water-cooling. All samples were observed under stereomicroscope (Carl Zeiss Axio Zoom V16, Jena,
Germany) at a magnification of $25 \times$ to $80 \times$ to determine incidence of dentinal cracks. Crack classification was explained at 4 levels;

1) No Defect (Score 0): Root dentin devoid of craze lines, complete cracks, and incomplete cracks.

2) Craze Line (Score 1): Line extending from outer surface into dentin but does not reach the canal lumen.

3) Partial or Incomplete Crack (Score 2): Line extending from the canal wall into the dentin withoutreaching the outer surface of the root.

4) Fracture or Complete Crack (Score 3): Line extending from root canal space all the way to outer surface of root.

\section{Statistical Analysis}

The incidences of defects between groups were statistically analyzed by Chi-square test at a significance level of $p<0.05$.

\section{RESULTS}

There was no statistical difference between all groups $(\mathrm{p}=0.361)$ while Adaptive motion showed fewer defects than rotary motion groups at all part of the roots. Adaptive motion groups have also showed no defect at the coronal part of the teeth either. Moreover, all groups had defects as compared with control group (Figure 1).

Complete or partial fracture formation was not observed in all the specimens examined. The specimens with craze lines were classified in Table 1. There was no statistical difference between all groups while Adaptive motion showed fewer defects than rotary motion groups at all part of the roots. Adaptive motion groups have also showed no defect at the coronal part of the teeth too. In addition all groups had defect as compared with control group.

No cracks were observed in the control group. In groups 2 and 4 no fracture was observed at the level of $9 \mathrm{~mm}$ and similarly in group 4 at $6 \mathrm{~mm}$ level. Group 3 showed a significantly higher dentin crack formation followed by Group 1, 2, 4 and control group respectively $(p<0.039)$. There was no significant difference between groups at $6 \mathrm{~mm}$ and $9 \mathrm{~mm}$ levels $(p=0.497)$ except for only $3 \mathrm{~mm}$ level $(p<0.035)$ (see Table 1). 


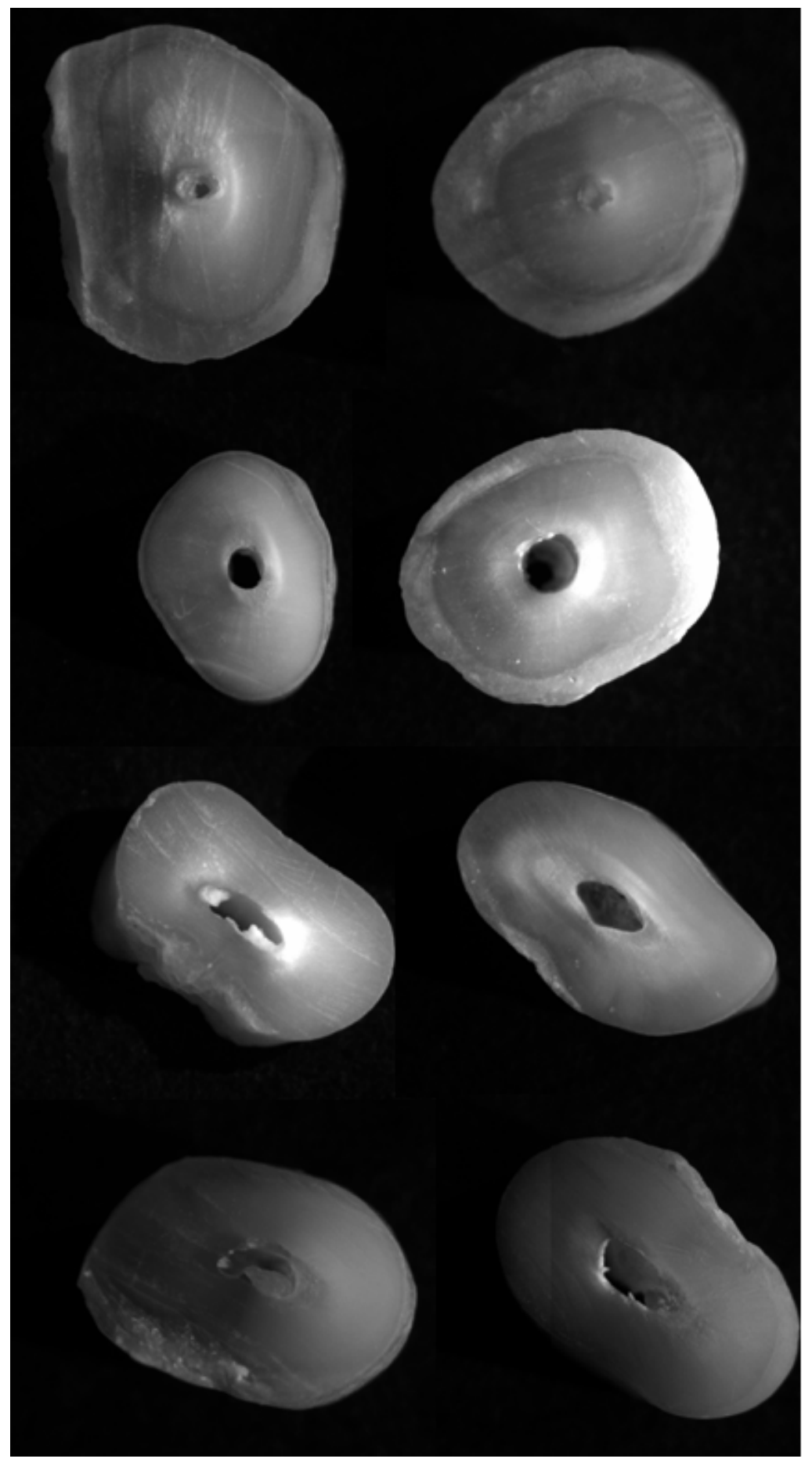

Figure 1. Craze lines at PT Next with adaptive motion (A), no defect at PT Next with adaptive motion (B), craze lines at PT Next with continuous rotation (C), no defect at PT Next with continuous rotation (D), craze Lines at TF with adaptive motion (E), no defect at TF with adaptive motion $(\mathrm{F})$, craze Lines at TF with continuous rotation $(\mathrm{G})$, no defect at $\mathrm{TF}$ with continuous rotation $(\mathrm{H})$. $\mathrm{PT}=$ ProTaper, $\mathrm{TF}=$ Twisted File 
Table 1. Incidence of dentinal crack formation in different root dentin levels

\begin{tabular}{lcccc}
\hline \multicolumn{4}{c}{ Absolute Number of Defects } \\
\hline Group 1 & $9 \mathrm{~mm}(\%)$ & $6 \mathrm{~mm}(\%)$ & $3 \mathrm{~mm}(\%)$ & Total defected roots per group (\%) \\
Group 2 & $3(15)$ & $3(15)$ & $3(15)$ & $9(15)$ \\
Group 3 & $5(25)$ & $2(10)$ & $2(10)$ & $4(7)$ \\
Group 4 & $0(0)$ & $5(0)$ & $3(15)$ & $13(22)$ \\
Control & $0(0)$ & $0(0)$ & $3(15)$ & $3(5)$ \\
$\boldsymbol{p}$ value & 0.497 & 0.497 & $0(0)$ & $0(0)$ \\
\hline
\end{tabular}

Group 1 = PT Next with Continuous Rotation, Group 2 = PT Next with Adaptive Motion, Group $3=$ TF Adaptive with Continuous Rotation, Group $4=\mathrm{TF}$ Adaptive with Adaptive Motion, PT $=$ ProTaper, $\mathrm{TF}=$ Twisted File

\section{DISCUSSION}

As a result of current instrumentation systems, obturation techniques and operator related factors, there is no an effective method to avoid craze lines, dentinal defects or vertical root fracture $[5,9]$. Endodontic treatments such as post space preparations, retreatment procedures or occlusal forces such as bruxism have a potential to create dentinal cracks too. In addition there is not certain evidence that craze lines produce complete dentinal cracks [12]. Previous studies have not been able to determine the exact role of reciprocal or rotary motion in the dentin crack formation. Burklein et al. [8] reported that at the apical level reciprocating motion produced more dentinal cracks than rotational systems. On the contrary, Liu et al. [10] showed that reciprocating files had better performance to produce dentin cracks. There is no definite consensus on this issue but in the present study, dentin defects were observed in all groups regardless of using file types and the NiTi files used at continuous rotation produced more dentinal defects on all root level.

Preliminary examination showed that any dentin defect was observed in extracted teeth. On the other hand it was very unacceptable that there was no crack after instrumentation at all groups. Unfortunately previous studies reported several complete dentin crack formation in similarly designed studies[5-8, 10 , $11,13,14]$. It can be explained that operator's skills and experiences are an effective factor for promoting crack formation and freshly extracted teeth are resistant to instrumentation. In addition it is clear that separation of crowns with diamond saw method is completely safe.

Dentin cracks studies have some limitation. The correlation between the present study method and clinical situation is questionable. For example patient age is an essential factor in the presence of dentinal cracks [13]. But we have no chance to determinate patient age of the extracted human molar teeth anyway. In addition using stereomicroscope should be questioned about dentinal cracks controlling at the same time.

Sectioning technique may be affected the defect formation. Because periodontal ligament absorb the occlusal forces and protect the dentinal wall against the crack formation. However no dentin defects observed in the control group,despite absence of periodontal ligament, indicated that the sectioning method was acceptable and safe. On the other hand extraction of the teeth is a major factor to promote dentin cracks. However stereomicroscope imaging using the present study may not be a suitable method to detect the micro-cracks. It is reported that optical coherence tomography and infrared thermography can be used in such studies to eliminated sectioningrelated defects in the future studies [15].

Another parameter that needs to be emphasized is the cross-section design of the NiTi Rotary files. According to the manufacturer, rectangular crosssectional design of PT Next instruments reduces the contact area between dentin and file which minimizes taper lock and screw effect while increasing cutting efficiency. This design also allows progressive tapers on the file production. Twisted File Adaptive NiTi 
rotary files have triangular cross section design that results lower cutting efficiency and less chip space that accumulates more dentin chip in the root canal complex. More stress accumulates on the root canal wall so dentinal defects may occur. Present study found that triangular designed NiTi file on rotation mode products more crack formation. However our results also showed that there is no significant difference on crack formation between the groups. It means that preparation technique is an important factor than file designs.

\section{CONCLUSION}

Under the limitations of the present study, root canal preparation with two rotary systems, both continuous rotation and reciprocating motion resulted in dentinal defects. At all thesections of the related samples, adaptive motion produced less dentinal defects (craze lines) than rotary groups. However there was no difference between adaptive motion and rotary motion statistically.Therefore the null hypothesis was accepted.

\section{Conflict of interest}

The authors disclosed no conflict of interest during the preparation or publication of this manuscript.

\section{Financing}

The authors disclosed that they did not receive any grant during conduction or writing of this study.

\section{REFERENCES}

[1] Young GR, Parashos P, Messer HH. The principals of techniques for cleaning root canals. Aust Dent J 2007;52(1 Suppl):S52-63.
[2] Wildey WL, Senia ES, Montgomery S. Another look at root canal instrumentation. Oral Surg Oral Med Oral Pathol 1992;74:499-507.

[3] Hulsmann M, Peters OA, Dummer PM. Mechanical preparation of root canals: shaping goals, techniques and means. Endod Topics 2005;10:30-76.

[4] Peters OA. Current challenges and concepts in the preparation of root canal systems: a review. J Endod 2004;30:559-67.

[5] Zandbiglari T, Davids H, Schafer E. Influence of instrument taper on the resistance to fracture of endodontically treated roots. Oral Surg Oral Med Oral Pathol Oral Radiol Endod 2006;101:126-31.

[6] Bier CA, Shemesh H, Tanomaru Filho M, Wesselink PR, Wu MK. The ability of different nickel-titanium rotary instruments to induce dentinal damage during canal preparation. J Endod 2009;35:236-8.

[7] Adorno CG, Yoshioka T, Suda H. The effect of root preparation technique and instrumentation length on the development of apical root cracks. J Endod 2009;35:389-92.

[8] Burklein S, Tsotsis P, Schafer E. Incidence of dentinal defects after root canal preparation: reciprocating versus rotary instrumentation. J Endod 2013;39:501-4.

[9] Ruddle CJ. Single-file shaping technique: achieving a gold medal result. Dent Today 2012;1:1-7.

[10] Liu R, Hou BX, Wesselink PR, Wu MK, Shemesh H. The incidence of root microcracks caused by 3 different single-file systems versus the ProTaper system. J Endod 2013;39:1054-6.

[11] Karataş E, Gündüz HA, Kırıcı DÖ, Arslan H, Topçu MÇ, Yeter KY. Dentinal crack formation during root canal preparations by the twisted file adaptive, ProTaper Next, ProTaper Universal, and WaveOne instruments. J Endod 2015;41:261-4.

[12] Shemesh H, van Soest G, Wu MK, Wesselink PR. Diagnosis of vertical root fractures with optical coherence tomography. J Endod 2008;34:739-42.

[13] Kansal R, Rajput A, Talwar S, Roongta R, Verma M. Assessment of dentinal damage during canal preparation using reciprocating and rotary files. J Endod. 2014;40:1443-6.

[14] Arias A, Lee YH, Peters CI, Gluskin AH, Peters OA. Comparison of 2 canal preparation techniques in the induction of microcracks: a pilot study with cadaver mandibles. J Endod 2014;40:982-5.

[15] Varela-Patiño P, Ibañez-Párraga A, Rivas-Mundiña B, Cantatore G, Otero XL, Martin-Biedma B. Alternating versus continuous rotation: a comparative study of the effect on instrument life. J Endod 2010;36:157-9. 